\title{
Aula interactiva 3D como recurso para la enseñanza en el Tecnológico Nacional de México campus Oaxaca
}

\section{Interactive classroom 3D as a resource for teaching at the Tecnológico Nacional de México campus Oaxaca}

\author{
ALTAMIRANO-CABRERA, Marisol†*, BENITEZ-QUECHA, Claribel, DIAZ-LARA, Carlos Alberto \\ y TORAL-ENRIQUEZ, Fernando
}

Tecnológico Nacional de México Campus Oaxaca

Tecnológico Nacional De México Campus Iguala

ID $1^{\text {er }}$ Autor: Marisol, Altamirano-Cabrera / ORC ID: 0000-0001-5800-9655 CVU CONACYT ID: 657390

ID $1^{\text {er }}$ Coautor: Claribel, Benitez-Quecha / ORC ID: 0000-0001-6516-5760

ID $2^{\text {do }}$ Coautor: Carlos Alberto, Diaz-Lara / ORC ID: 0000-0002-1782-1900

ID $3^{\text {er }}$ Coautor: Fernando, Toral-Enriquez / ORC ID: 0000-0002-5144-8839

DOI: $10.35429 /$ JCT.2019.10.3.9.16

Recibido 19 de Julio 2019, Aceptado, 05 de Septiembre, 2019

\section{Resumen}

En este artículo se construye un prototipo de aula virtual inmersiva implementado en el I.T.Oaxaca que sirve de apoyo al proceso de aprendizaje de los estudiantes de la Ingeniería en Sistemas Computacionales. Sin descartar a futuro se incorpore esta plataforma de asesorías a otras ingenierías que se ofertan en la institución. La propuesta experimental utiliza el metaverso de Second Life, simulando espacios físicos en tres dimensiones y que permiten a los usuarios (avatares) interactuar entre sí con la finalidad de intercambiar información y experiencias. A pesar de estar en la fase beta de la implementación, se han obtenido indicadores interesantes y expectativas de aprendizaje en los estudiantes al asociar el conocimiento mediado por la interacción entre sus avatares y la escenografía 3D; el cual sumerge al estudiante en una nueva aventura de estudio con altos niveles de interactividad, conduciéndolo de una manera informal a un nuevo estilo de aprendizaje.

\section{Avatar, Metaverso, Mundos virtuales}

\begin{abstract}
This article builds a prototype of an immersive virtual classroom implemented in the I.T.Oaxaca that serves as support to the learning process of the students of Computer Systems Engineering. Without discarding in the future, this advisory platform will be incorporated into other engineering services offered at the institution. The experimental proposal uses the metaverse of Second Life, simulating physical spaces in three dimensions and allowing users (avatars) to interact with each other in order to exchange information and experiences. Despite being in the beta phase of the implementation, interesting indicators and learning expectations have been obtained in the students by associating the knowledge mediated by the interaction between their avatars and the 3D scenography; which immerses the student in a new study adventure with high levels of interactivity, leading it in an informal way to a new learning style.
\end{abstract}

Avatar, Metaverse, Virtual worlds

\footnotetext{
* Correspondencia del Autor (Correo electrónico: marisol_altamirano@ hotmail.com)

$\dagger$ Investigador contribuyendo como primer autor.
} 


\section{Introducción}

Con el beneficio que implican las TIC en el proceso de enseñanza aprendizaje y los avances tecnológicos con impacto social como son el procesamiento en la nube, la Realidad Aumentada (AR) y Realidad Virtual (VR) para crear entornos más sofisticados y atractivos para los actores en un aula; quienes mediante interacción podrán desarrollar actividades académicas orientadas a perfeccionar las habilidades, competencias y capacidades de los estudiantes mejorando el proceso de enseñanza aprendizaje en todo el sistema.

Esteve,F. y Gisbert,M. (2013) indican que con estos instrumentos, la principal competencia que se desarrolla es la digital, entendida como "El conjunto de habilidades y conocimientos tecnológicos, informacionales, comunicativos y multimedia que cualquier estudiante debe desarrollar y utilizar, de manera creativa, crítica, reflexiva, ética, y efectiva". El desarrollo y la evaluación de competencias, como parte intrínseca del proceso de enseñanzaaprendizaje, requiere de situaciones y estrategias formativas adecuadas, no sólo centradas en la adquisición y memorización de conceptos y contenidos sino en la puesta en acción de dichos conocimientos, junto con habilidades, destrezas $\mathrm{y}$ actitudes, de manera efectiva y eficiente, que se magnifica con el uso diario de herramientas electrónicas como los teléfonos celulares, tabletas, equipos de cómputo, Internet, los videojuegos, etc.

Estos dispositivos emplean entornos virtuales que son espacios que permiten realizar una inmersión en un entorno simulado, interactuar con los otros usuarios, utilizar, crear e intercambiar objetos, y son personalizables y programables, según la finalidad deseada. Estos entornos tienen muchas potencialidades en el campo educativo, tanto para la realización de experiencias y prácticas formativas, como para actividades de experimentación, pruebas, simulaciones o actividades de trabajo en grupo, quedando registrada toda la actividad realizada en ellas, de manera rigurosa y sistemática que repercute tanto en el rendimiento académico como en la motivación de los estudiantes.
Al emplear estrategias innovadoras con la creación de entornos virtuales, el docente podrá recrear su clase e intercambiar ideas de manera abierta y responsable, también podrá integrar las actividades propuestas dentro de un curso específico, evaluarlas y darles el seguimiento que corresponde; con lo anterior, el eje académico se ve fortalecido con múltiples entornos de aprendizaje, los cuales incluyen plataformas y recursos educativos digitales, laboratorios virtuales, redes sociales $\mathrm{y}$ escenarios virtuales que permiten que el aprendizaje sea ubicuo.

Actualmente el $75 \%$ de los docentes del departamento de sistemas y computación del Instituto Tecnológico de Oaxaca se apoya de plataformas LMS siendo Moodle la más usada, redes sociales y/o correo electrónico para la comunicación, envío y revisión de actividades, así como para publicar material de estudio. La limitante de estas herramientas es su falta de dinamismo, ya que generalmente ofrecen solamente la creación de foros y asignación de actividades.El proyecto Aula Interactiva en 3D se propone como una herramienta de apoyo académico para docentes y estudiantes de la institución que ofrecerá beneficios como: mayor comunicación fuera del horario de clases, la realización de actividades de refuerzo académico, permitiendo realizar simulaciones de actividades comunes en un ambiente controlado. El documento que se presenta está organizado en secciones que abarcan desde la fundamentación teórica que orientaron el desarrollo del aula virtual, las actividades realizadas durante las fases definidas por la metodología de desarrollo de software empleada (XP); integrado en tres iteraciones dentro del proyecto. Cada una de estas fases, a su vez incluyen las etapas de: análisis, diseño, codificación y pruebas.

\section{Planteamiento del problema}

Desde la creación del TecNM, el 14 de julio del 2014, los trabajos en el área de docencia se están orientado a perfeccionar las habilidades y capacidades de los estudiantes con el apoyo de las Tic's, las cuales mejoran el proceso de enseñanza-aprendizaje en todo el sistema. Con lo anterior, el eje académico se ve fortalecido con múltiples entornos de aprendizaje, los cuales incluyen plataformas y recursos educativos digitales, laboratorios virtuales, redes sociales y escenarios virtuales, entre otros. 
Desafortunadamente, una de las condiciones que no permiten la inclusión de estas herramientas en el TecNM campus I. T. Oaxaca, son los costos de las licencias que por ser altos no se autorizan por el órgano central y el poco interés que demuestran los docentes para fortalecer sus competencias, hacia el uso de tecnologías para hacer que el aprendizaje sea ubicuo.

Según el registro del Sistema Integral de Información (SII,2018) el TecNM campus I. T. Oaxaca se encuentran registrados 300 docentes, con al menos 20 horas frente a grupo por semana cada uno, de los cuales el $82 \%$ utiliza el proyector y equipo de cómputo para impartir los temas de sus asignaturas, en ocasiones con material realizado por ellos en algún software de presentación o documentos descargados directamente de alguna página de internet y/o Ambientes Virtuales de Aprendizaje. Mientras que el $18 \%$ restante continúan impartiendo clases de manera tradicional con el apoyo de un pintarron.

Los métodos que la mayoría de los docentes utilizan para impartir sus clases, a gran parte del alumnado le llega a generar aburrimiento y cansancio, que trae como consecuencia la falta de interés e inasistencia, sobre todo en materias consideradas en el Sistema De Asignación Y Transferencia De Créditos Académicos (SATCA) como teóricas, en las cuales existe poca interacción entre el estudiante y el docente y obviamente todo el proceso de seguimiento y evaluación es realizado de forma manual.

Otro factor influyente es el horario laboral y la disponibilidad con la que cuentan los profesores, el cual no les permite asesorar a todos sus estudiantes, a quienes de forma individual, tiene que evaluar: rúbricas, portafolios de evidencias, exámenes, competencias, entre otras. La plataforma Moodle, utilizada por los docentes que implementan ambientes virtuales de aprendizaje, tiene como objetivo principal ayudarles a crear comunidades de aprendizaje en línea, promueve una pedagogía constructivista social (colaboración, actividades, reflexión, critica), brinda al profesor el control total sobre todo el curso.
Siendo su principal desventaja el desarrollo de las actividades, ya que pueden ser mecánicas, dependiendo del diseño instruccional, generalmente en este tipo de herramientas sólo se pueden crear foros de información, enviar y recibir archivos. De esta manera al no existir mayor interacción entre los participantes, genera un bajo nivel de acceso.

Para disminuir esta problemática se propone un Aula interactiva 3D como herramienta de apoyo virtual que coadyuve con su formación académica.

\section{Objetivos}

- Identificar si el estudiante cuenta con las condiciones viables para acceder a un aula virtual e implementarla en el TecNM, campus I. T. Oaxaca.

Comprobar que un aula virtual será aceptada por los estudiantes del TecNM campus I. T. Oaxaca, en un $80 \%$, como recurso digital interactivo alineado a la ruta de aprendizaje.

- Aplicar la metodología XP para el desarrollo del proyecto.

- Construir el aula con una interfaz amigable y fácil de usar para el usuario.

Realizar pruebas con el usuario.

Obtener resultados

\section{Desarrollo}

El proyecto se desarrolla siguiendo las fases consideradas en la Metodología XP. Este método permite que todo el sistema, o algunas de sus partes, se construyan rápidamente para comprender con facilidad y aclarar ciertos aspectos en los que se aseguren que los implicados estén de acuerdo en lo que se necesita así como también la solución que se propone para dicha necesidad. En cada iteración se implementan las 4 etapas indicadas en el apartado anterior, el cual se va retroalimentando de los anteriores hasta mejorarlo. Los expertos creen que adaptarse a los cambios de requisitos en cualquier punto de la vida de un proyecto es una aproximación más realista que intentar definir todos los requisitos al inicio del proyecto e invertir esfuerzos después en controlar los cambios en los requisitos. 
Las etapas y procesos considerados para la consecución del proyecto se describen en las siguientes iteraciones:

\section{Primera Iteración}

\section{Análisis}

La primera fase consistió en la delimitación de los objetivos esperados, así como la identificación de los alcances y las limitaciones del proyecto. Una vez definido lo anterior Se realizó una investigación para comparar entre las diferentes opciones disponibles para la implementación de un aula virtual. Las opciones analizadas fueron tres: OpenSim, OpenGL y Second Life.

\section{OPENSIM}

Contracción de Open (abierto) Simulator (simulador) es un servidor 3D de código abierto que permite crear ambientes virtuales o mundo virtual a los que se accede a través de una gran variedad de visores (clientes) o protocolos (software y web). Configurable para suplir las necesidades del propietario del mundo virtual y puede ser extendido usando módulos. La licencia de OpenSim es BSD, código libre. Se trata de una plataforma para controlar un mundo virtual y soporta múltiples e independientes regiones conectadas entre sí. Con este recurso es posible poner en marcha un mundo virtual en un servidor Web y enlazarlo a través de Internet con otros mundos virtuales. También puede ser usado para crear un Grid privado, como si fuera una intranet.

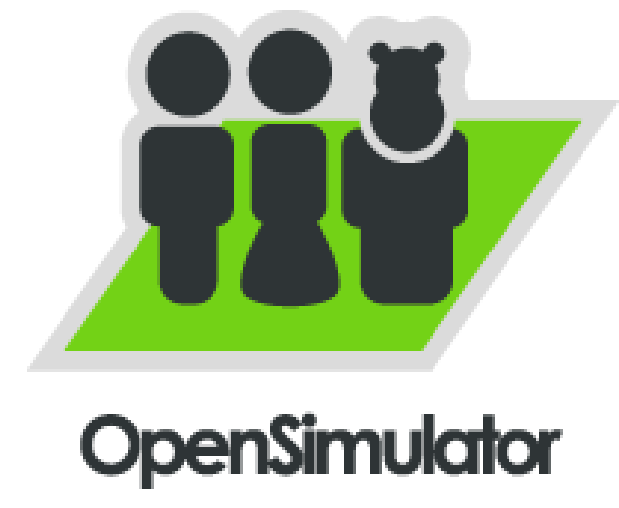

Figura 1 Logo de OpenSimulator Fuente:openSim.org

\section{OPENGL}

OpenGL (Open Graphics Library) es una especificación estándar que define una API multilenguaje y multiplataforma para escribir aplicaciones que produzcan gráficos $2 \mathrm{D}$ y $3 \mathrm{D}$. La interfaz consiste en más de 250 funciones diferentes que pueden usarse para dibujar escenas tridimensionales complejas a partir de primitivas geométricas simples, tales como puntos, líneas y triángulos. Fue desarrollada originalmente por Silicon Graphics Inc. (SGI) en 19922 y se usa ampliamente en CAD, realidad virtual, representación científica, visualización de información y simulación de vuelo. También se usa en desarrollo de videojuegos, donde compite con Direct3D en plataformas Microsoft Windows.

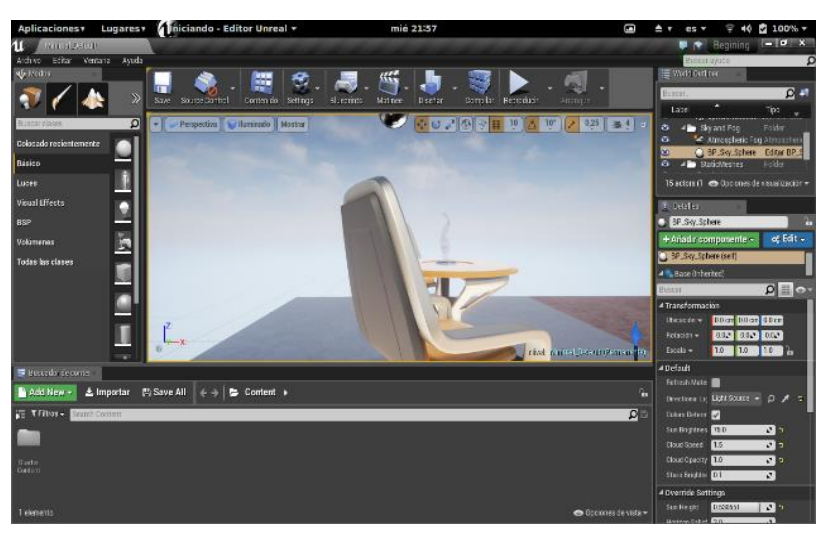

Figura 2 Entorno de desarrollo de OpenGL Fuente Elaboración Propia

Debido a sus características, esta fue una opción inviable debido a la necesidad de interconexión de una gran cantidad de usuarios a la vez, la cual es una de las debilidades de OpenGL.

\section{SECOND LIFE}

La opción más viable fue Second Life, debido a que se puede utilizar en las plataformas más utilizadas como son Windows y OSx, además, cuenta con los mejores gráficos de las tres existentes y una plataforma consolidada $y$ respaldada por una amplia comunidad de usuarios. 


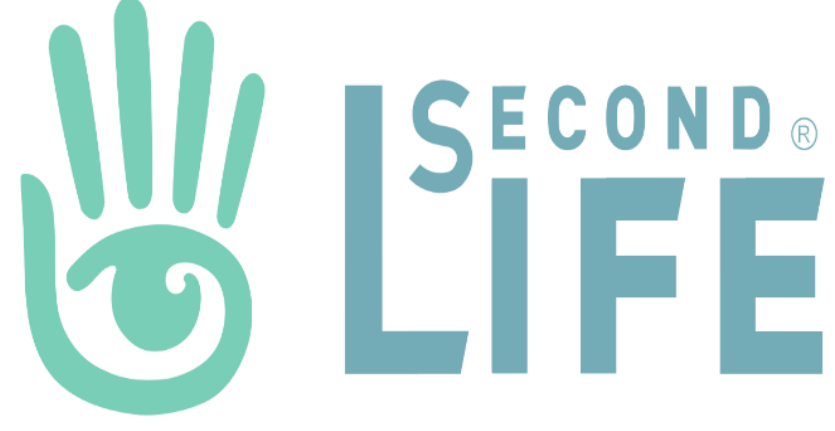

Figura 3 Logo de Second Life

Fuente https://yt3.ggpht.com

Dado que Second Life es una plataforma con licencia privativa, propiedad de Linden Lab, se requiere cubrir un costo por el acceso a algunas funcionalidades(cuenta premium), el cual se incluye un terreno básico asignado al residente y además permite la construcción y adquisición de objetos, modificación de los espacios, así como programación de scripts usando Linden Scripting Language. los cuales permiten además el acceso a las API's de Second Life para incluirlas y aprovecharlas desde un sitio web propio, administrando así el registro, acceso e información de los usuarios del aula virtual.

\section{Diseño}

Una vez seleccionada la plataforma, para la realización del aula virtual se realizó un recorrido por el mundo virtual de Second Life para identificar las características de algunos espacios destinados a actividades de aprendizaje $\mathrm{y}$, de este modo, tomarlos como referencia para el futuro desarrollo propio.

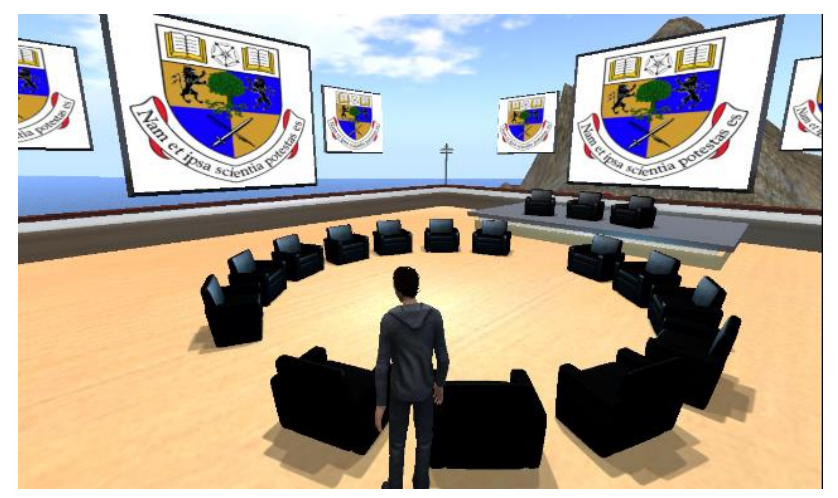

Figura 4 Entorno de Second Life Fuente second life.com
Se identificaron características deseables del aula virtual, adecuándose a las limitantes de espacio correspondientes al terreno que se incluye con una cuenta premium, tales como, buena iluminación, asientos bien distribuidos, presentadores de diapositivas y un espacio común para la interacción libre.

\section{Codificación}

Como primer paso de codificación se realizaron scripts simples utilizando Linden Scripting Language que permiten a los usuarios realizar acciones básicas como sentarse de forma definida en algún lugar y variar la posición del avatar. Así mismo se investigó la estructura, sintaxis y características fundamentales del lenguaje. Se desarrolló el prototipo inicial del aula virtual, con los objetos básicos para la interacción de los usuarios, simulando un entorno de la vida real. Para esto se utilizaron las herramientas incluidas dentro del visor de second life.

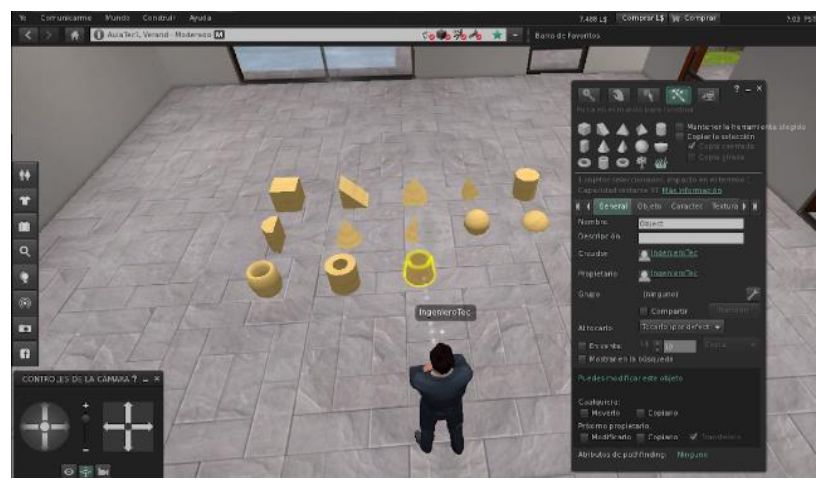

Figura 5 Formas primitivas (prims)

Fuente Elaboración Propia

\section{Pruebas}

Para comprobar las capacidades de conectividad e interacción que brinda el aula virtual se realizaron pruebas de uso, en un primer momento, con las cuentas de usuario destinadas para tal fin, sin embargo, para verificar el soporte para un número más elevado de usuarios y el correcto funcionamiento de la plataforma utilizada en dichas circunstancias, se solicitó el apoyo a 93 estudiantes distribuidos en 3 grupos para que ingresaran al mismo tiempo y probaran la funcionalidad de ese espacio virtual. 
Como resultado de estas primeras pruebas, se corroboró que la funcionalidad del aula no se ve afectada por el número de usuarios conectados en el mismo espacio, se validaron los sistemas de comunicación como el chat y la voz bidireccional. Aunque en éste último aspecto, se reportaron por parte de los usuarios algunas dificultades para la configuración de los micrófonos, lo cual fue enlistado en las necesidades para corrección de errores. Se realizaron 3 iteraciones más, en comunión con los usuarios finales (profesores y estudiantes), agregando un módulo exclusivo para profesores en el que podrán dar seguimiento a los estudiantes que requieran el apoyo, ingresando mediante una cuenta apuntes, presentaciones y ejercicios que podrán ser evaluados y resueltos al mismo tiempo.

\section{Cuarta Iteración}

\section{Análisis}

El Aula Virtual es completamente funcional destacando los siguientes aspectos a mejorar:

\section{- $\quad$ Pizarrón Interactivo \\ - $\quad$ Texturas \\ - Decoración Interior \\ - Decoración Exterior}

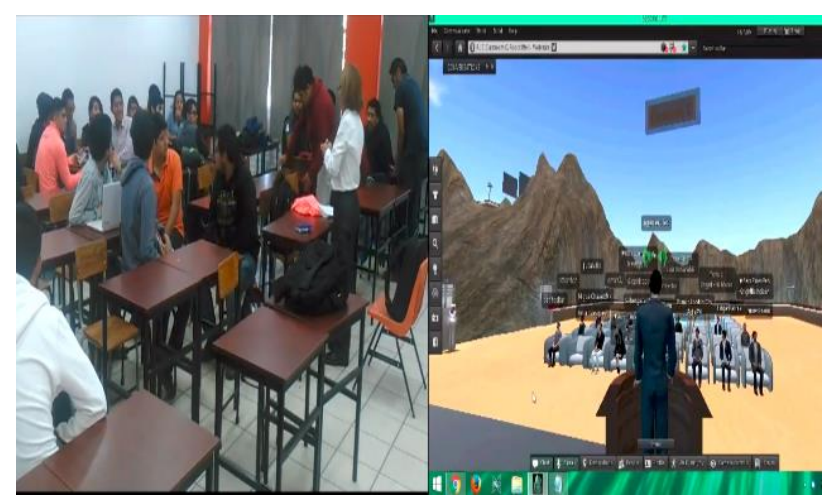

Figura 6 Catedra Impartida por profesora Fuente Elaboración Propia

\section{Diseño}

El pizarrón debe ser fácil de interactuar con él, de forma que cumpla la función de un pizarrón en el mundo real.

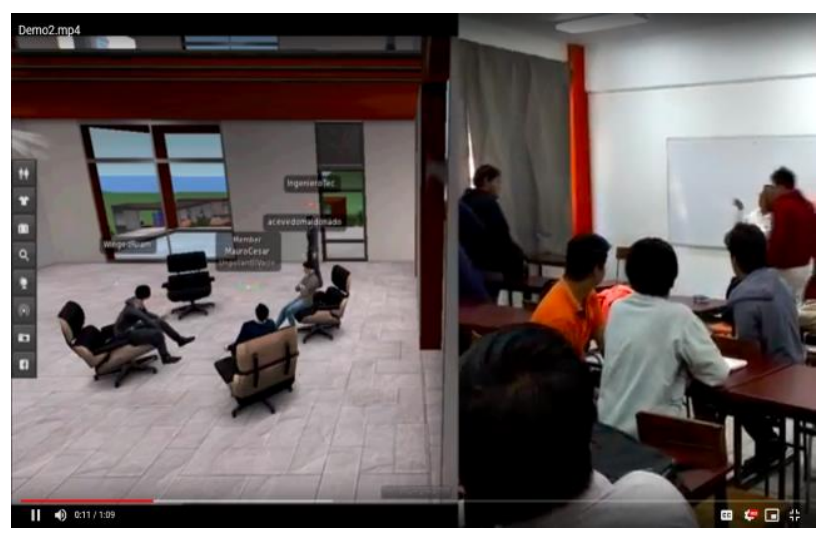

Figura 7 Prototipo de Pizarrón

Fuente Elaboración Propia

\section{Pruebas}

Como etapa final del proyecto se realizaron pruebas integrales del funcionamiento del aula virtual, en sus diversos apartados, retomando las observaciones detectadas en las primeras iteraciones para asegurar que la funcionalidad fuera completa y no presentara inconvenientes, tanto para el usuario final como para los desarrolladores que deseen involucrarse en la comprensión y mantenimiento del proyecto.

Para la realización de dichas pruebas, se diseñó el curso de nivelación de fundamentos de programación que se imparte a los estudiantes de nuevo ingreso. Para el año 2018 se integraron 6 grupos; los cuales 3 llevaron de manera presencial la materia durante 20 días en un periodo del $2^{\circ}$. Al 27 de Julio del 2018 y los otros 3 grupos la cursaron de manera virtual, con un tutor asignado y cada estudiante desde la comodidad de su casa o lugar elegido. Se requería en este caso, equipo de cómputo y conexión a internet.

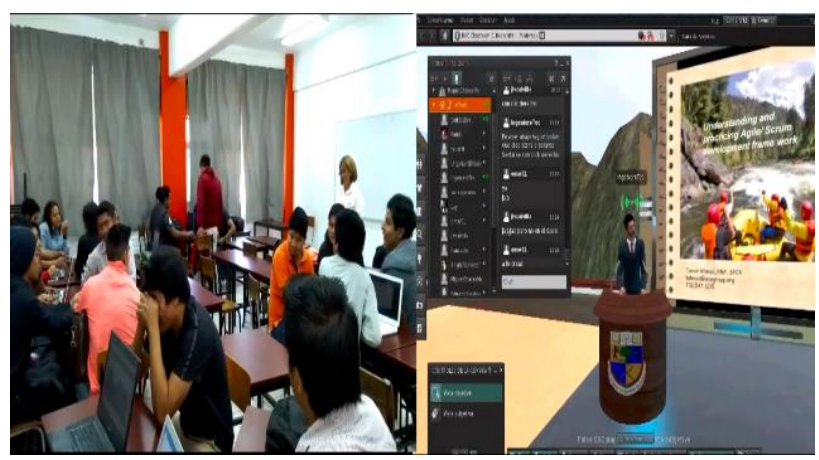

Figura 8 Aula virtual VS Aula física Fuente Elaboración Propia 


\section{Resultados}

Los estudiantes participantes en el curso de nivelación de manera virtual fueron elegidos principalmente por su lugar de origen, se tiene el indicador de que el $43 \%$ de los estudiantes que cursan una licenciatura en la institución son foráneos y a ellos se les pidió su autorización para participar en las pruebas de este proyecto, proponiéndolo como una opción para evitar un gasto extra. Además que en esas fechas muchos no terminan aún su educación media superior. Obviamente el hecho de no tomarlo presencial no significaría que no atenderían la clase diaria de 3 horas, en la cual el profesor impartía la clase, generaba diversas actividades, foros $\mathrm{y}$ retroalimentaba a los estudiantes. Como resultado de estas pruebas, se comprobó la efectividad del aula virtual para lograr los cometidos que se plantearon, del mismo modo, se concluye que la interacción entre los usuarios, así como entre usuarios y objetos, se lleva a cabo de manera acorde con lo previsto

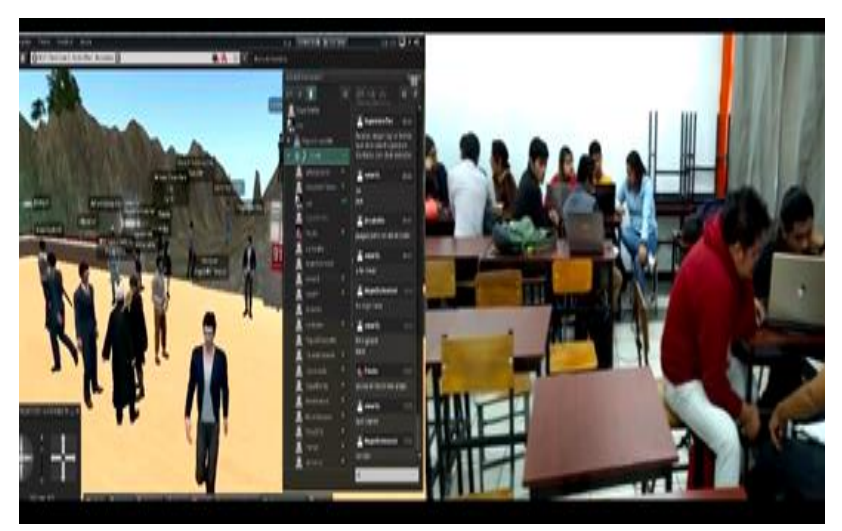

Figuran 9 Actividades en clase Fuente Elaboración Propia

\section{Agradecimientos}

Agradecemos al Tecnológico Nacional de México campus Instituto Tecnológico de Oaxaca e Instituto Tecnológico de Iguala, las facilidades otorgadas para la realización del proyecto. Principalmente a los estudiantes y egresados que nos apoyaron con su experiencia y tiempo.

\section{Conclusiones}

El uso del aula virtual 3D fomenta el trabajo colaborativo entre los estudiantes y mejora la interacción maestro-estudiante al generarse una experiencia enriquecedora; ya que el aula virtual permite:
- Ejercitar y evaluar habilidades interpersonales a distancia.

- Realizar actividades colaborativas

- Igualar a los participantes sin distinción de sus características físicas

- Interactuar simultáneamente a cientos de participantes desde cualquier ubicación.

- $\quad$ Promover el uso de nuevas tecnologías

Con los cuestionarios aplicados se reflejó un nivel aceptación del $99 \%$ en el uso del Aula Virtual por parte de los estudiantes, por lo que la hipótesis es aceptada. El Aula Virtual tiene un gran potencial de crecimiento, ya que con la adquisición del estatus de Educador en Second Life se puede crear todo un campus virtual. El alcance a futuro de este proyecto no solo se puede quedar como un campus virtual que emule una universidad del mundo real, más allá de eso, el desarrollo de objetos programados con funciones atractivas puede ser una gran fuente de ingresos.

\section{Referencias}

Bereiter, C. y Scardamalia, M., (1987): Psychology of written composition. Hillsdale N. J., Lawrence Erlbaum.

Dolan S. y Martín, I. (2002): Los 10 Mandamientos para la Dirección de personas, Barcelona Ed. Gestión 2000

Esteve, F. y Gilbert, M. (2013). Competencia digital en la educación superior: instrumentos de evaluación y nuevos entornos. Enlace Revista Venezolana de información, Tecnología y Conocimiento, 10(3),29-43.

García-Quintanilla M. y Casarini M. (2009). La tecnología para el cambio educativo: Reflexiones y experiencias. UANL. Monterrey. México

Horton, W. (2000) Designing web based training Wiley Computer Publisher, New York, NY.

Jara Ignacio y Toledo Castor. (2009) Portales educativos. En "Las Tecnologías de la Información y la Comunicación en el aula". Unesco. Plan Ceibal. Mec. Uruguay. 
Nosnik, A. (2005) Las comunicación productiva: Un nuevo enfoque teórico. Las teorías de la comunicación. Número 34.Tomado de www.razonypalabra.org.mx

Rabajoli Graciela y Mario Ibarra (2008) Características de un recurso educativo para cumplir su objetivo.

Rodríguez, T. y Baños, M.(2011) E-learning en mundos virtuales 3D. Una experiencia educativa en Second Life. Revista Icono14 [en línea] 1 de julio de 2011, Año 9, Volumen 2. pp. 39-58. Recuperado el 27 de Febrero de 2019, de http://www.icono14.net.

Rowell, A., (1997) Virtual Reality, Computer Graphics World.

Scagnoli, N. (2010). El aula virtual: usos y elementos que la componen. Universidad de Illinois, Urbana. Publicado en CEDIPROE.

Siliceo A. Alfonso. (2014) Capacitación y desarrollo de personal cuarta edición, Ed. Limusa, México.

SII (2018. Sistema integral de información. Tecnológico nacional de México

UNESCO. (2013-2014) Informe de Seguimiento de la Educación para Todos en el Mundo. 